\title{
Design and Implementation of LZW Data Compression Algorithm
}

\author{
Simrandeep kaur, Student ${ }^{1}$; V.Sulochana Verma,Project Consultant ${ }^{2}$ \\ Academic and Consultancy Services Division \\ C-DAC Mohali, Punjab India \\ Email: simrandeepkaur25@yahoo.com1; Email: suchivlsi@gmail.com2
}

\begin{abstract}
LZW is dictionary based algorithm, which is lossless in nature and incorporated as the standard of the consultative committee on International telegraphy and telephony, which is implemented in this paper. Here, the designed dictionary is based on content addressable memory (CAM) array. Furthermore, the code for each character is available in the dictionary which utilizes less number of bits (5 bits) than its ASCII code. In this paper, LZW data compression algorithm is implemented by finite state machine, thus the text data can be effectively compressed. Accurate simulation results are obtained using Xilinx tools which show an improvement in lossless data compression scheme by reducing storage space to $60.25 \%$ and increasing the compression rate by $30.3 \%$.
\end{abstract}

\section{Keywords}

Compression rate, LZW codes and Binary text.

\section{Introduction}

Data compression is often referred to as coding, where coding is general term showing any special representation of data which satisfies a given need. Information theory is defined as the study of efficient coding. Data compression may be viewed as a branch of information theory in which the primary objective is to minimize the amount of data to be transmitted. Data compression has an important role in the area of transmission and storage. It plays a key role in information technology. The reduction of redundancies in data representation in order to decrease data storage requirement is defined as data compression. It used less usage of resources such as memory space or transmission capacity. Data compression is classified as lossless and lossy compression. Lossless compression is used for text and lossy compression for image.

In 1980, Terry Welch invented LZW algorithm which became the popular technique for generalpurpose compression systems. It was used in programs such as PKZIP as well as in hardware devices. Lempel-Ziv-Welch proposed a variant of LZ78 algorithms, in which compressor never outputs a character, it always outputs a code. To do this, a major change in LZW is to preload the 
dictionary with all possible symbols that can occur. LZW compression replaces string of characters with codes. LZW algorithm is a lossless data compression algorithm which is based on dictionaries [1]. This LZW compressor maintains records with characters that have been read from a file to be compressed. Each character is represented by an index number in the dictionary. In this paper, we proposed a improve scheme for data compression. By utilizing, content access memory dictionaries are built in the proposed system. Each character in dictionary is replaced with a code which is less number of bits than its ASCII code. The proposed LZW algorithm is evaluated by finite state machine technique in VHDL. This paper is organized as follow in section 2 an introduction to LZW algorithm is explained; in section 3, LZW data compression algorithm using finite state machine (FSM) is described. In section 4 experiments and results are shown. Finally conclusion is exposed in section 5.

\subsection{Data Compression Model}

The block diagram of data compression model is described in figure 1 .

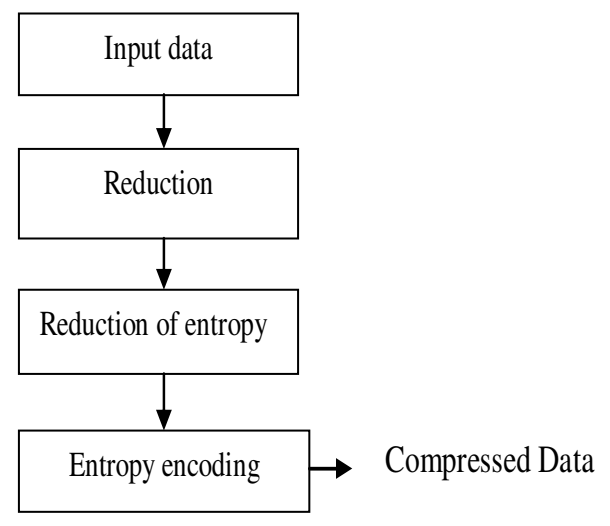

Figure 1: Data compression model

A data compression model consists of three major stages which are redundancy, reduction in entropy and entropy encoding.

\section{Data Compression Algorithm: LZW (Lempel-Ziv Welch) Algorithm}

There are many algorithms which have been used for data compression like Huffman and LempelZiv-Welch (LZW), arithmetic coding.LZW algorithm is the most popular algorithm. LZW algorithm is just like a greedy approach and divides text into substrings. Like the LZW algorithm proposed in [2]. LZW algorithm has both compression and decompression techniques which is explained as below.

\subsection{LZW Compression Algorithm}

LZW compression algorithm is dictionary based algorithm which always output a code for a character. Each character has a code and index number in dictionary. Input data which we want to 
compress is read from file. Initially data is entered in buffer for searching in dictionary to generate its code. If there is no matching character found in dictionary. Then it will be entered as new character in dictionary and assign a code. If character is in dictionary then its code will be generate. Output codes have less number of bits than input data. This technique is useful for both graphics images and digitized voice.

String j, char c;

j- get input character

while (there is still input character)

ch- transfer input string to $c h$.

if (ch is in dictionary)

Generate its codeword;

else

update ch and get next character to ch and

again search data in dictionary;

if ( it is not present in dictionary) then

add that string to dictionary;

end if;

Compression example: consider a string "BAABAABB" is given to LZW algorithm. Figure 2 shows the steps done by LZW to generate the output code is "1211211C". In following example when input string (BAABAABBC) is given as a text to LZW compression algorithm. Initially every single character will save in buffer. When ' $\mathrm{B}$ ' is move to buffer "parse string" then it will replace by 1. Character has its own ASCII code of 7 bit. In case of B, it has 65 as ASCII code. But in dictionary it will replace by 1 . So, less number of bits will be used to represent character. Similarly, AA will move forward and generating its code which is also fewer bits than original. BAA is saved in buffer its code is generated from both AA and B's codeword that is defined as 12. At last when full string has been searched in dictionary then its output will be generated as $1211211 \mathrm{C}$.

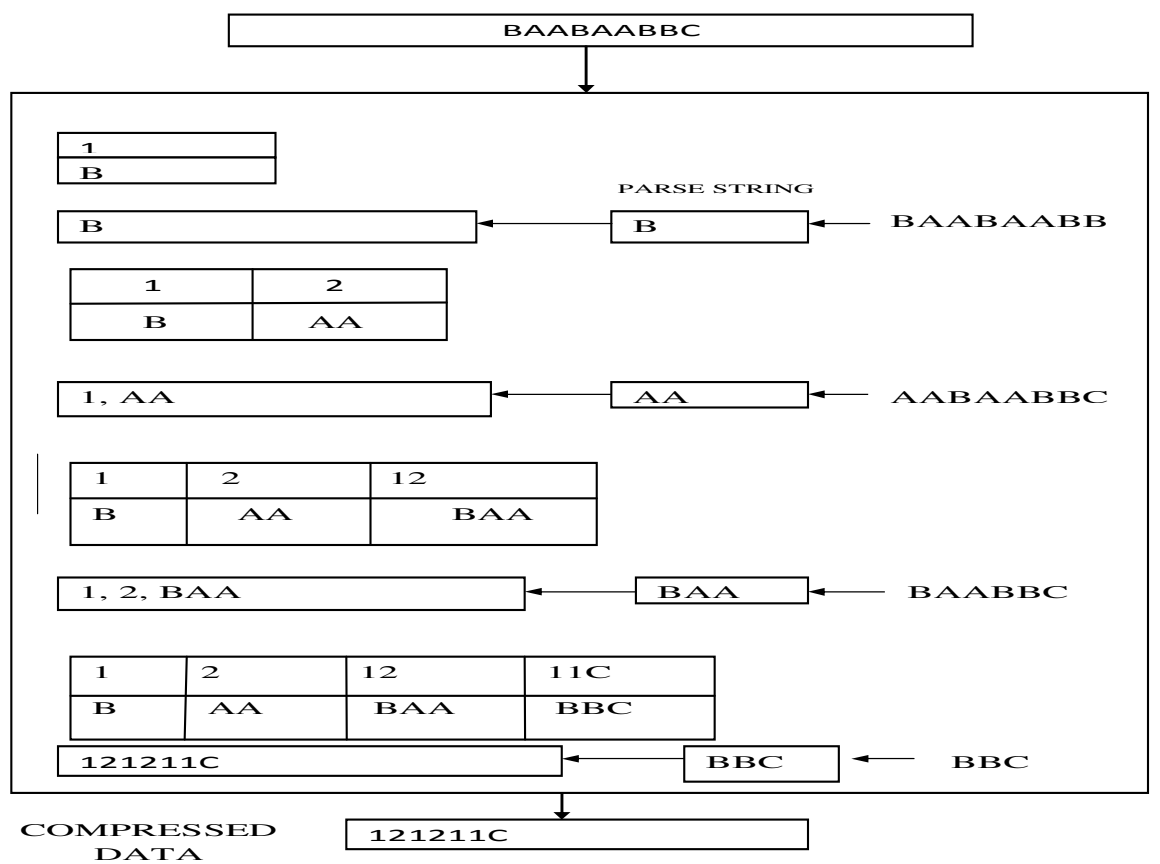

Figure 2: Example of LZW algorithm 


\subsection{LZW Decompression Algorithm}

In LZW decompression algorithm, it needs to take the stream of code output from the compression algorithm, and use them to exactly recreate the input stream. Decompression algorithm is shown as:

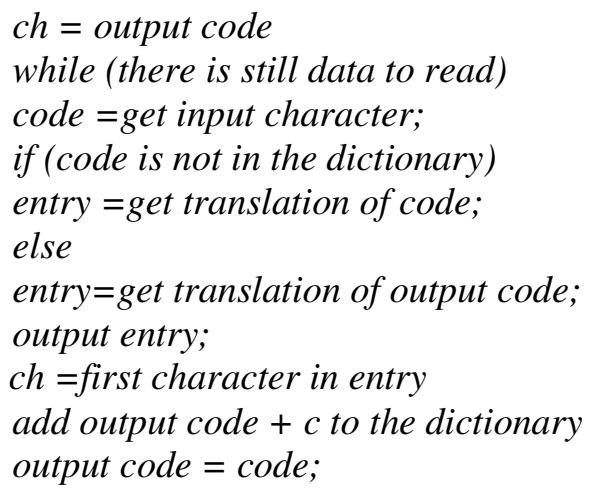

In decompression algorithm, code will be searched in dictionary and its character will be output.

\section{Implementation of LZW Algorithm}

The proposed finite state machine diagram of LZW algorithm is shown in figure 3.

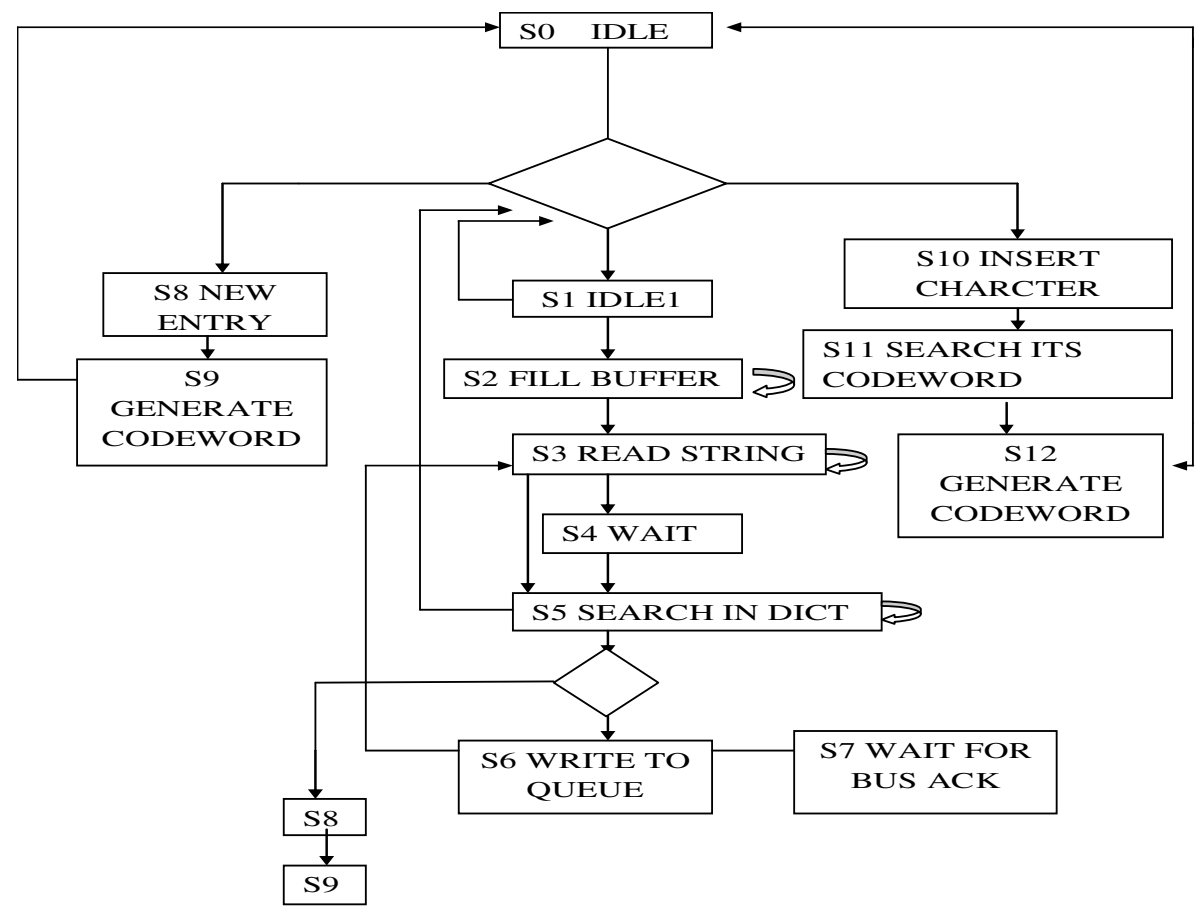

Figure 3: Finite state machine Diagram of LZW algorithm 
LZW algorithm initially has idle state. New character has been added to dictionary when no longer match will found in search process. LZW algorithm is execute state S8 for performing adding operation in dictionary. Dictionary is based on content access memory technique which has both content as well as code in it. Content access memory is special type of memory used for fast accessing data from memory. In the proposed system, initialization of compression signal is done before to perform LZW algorithm. Input data is entered to LZW algorithm through file. The proposed algorithm shifted whole input data to buffer which is defined in S3 state. Every single character has been searched in content access memory. If match signal is ' 1 ' then character was found in dictionary. Then code is transmitted to output buffer "de11". LZW decompressor must construct same steps like compressor. Decompressor has reviewed same process since it is possible to have input codes for searching in dictionaries to recreate its original string. Individual character's code can be also viewed in dictionary.

Table 1: Specifications of FSM state for LZW Algorithm

\begin{tabular}{|l|l|}
\hline \multicolumn{1}{|c|}{ State } & \multicolumn{1}{c|}{ Description } \\
\hline S0 idle & Initial state reset the system \\
\hline S1 idle1 & Initialization of signal \\
\hline S2Fill buffer & Transfer text from file to buffer \\
\hline S3Read string & Read character by character for searching \\
\hline S4 wait & For waiting \\
\hline S5 search in dict & For searching in dictionary by signal character \\
\hline S6Write to queue & Save output to output buffer \\
\hline S7 wait for ack & Wait for Bus acknowledge \\
\hline S8New Entry & Adding new entry \\
\hline S9Generate codeword & To generate codes \\
\hline S10 & Insert single character \\
\hline S11search its codeword & Check in dictionary \\
\hline S12 generate codeword & Display codeword \\
\hline Decompression & For performing decompression \\
\hline
\end{tabular}

\subsection{Improvement of the dictionary storage method}

LZW algorithm is mainly used for compressing character but not numeric. Every character has ASCII code which is of 7 bits. But in our proposed algorithm we have to replace character with 5 bit code in dictionary to improving data compression rate.

\section{Experimental Results}

LZW Compression algorithm is modelled in VHDL. The syntax of the RTL design is checked by using Xilinx tool. 


\subsection{Simulation Results}

In the proposed work, the simulations results are done using Xilinx ISE Simulator. Simulation results show an improvement in lossless data compression scheme. In addition to this, the proposed technique results in reduced storage space by $60.25 \%$ and increased compression rate by $30.3 \%$.

\subsection{LZW Compressor Result}

Figure 4 shows that input is given to LZW Compressor through text file. "Connect the input to logic one \& two \& three++*"string is entered to it. Input string having collection of special characters, alphabets. Whole text will transfer to buffer "data read "when data_write=1, load=1, clear $=1 . R d \_b=0, w r \_b=1$, data_write $=0$ and $1 z w \_s e a r c h=1$ are given to start searching process to find longest match in content access memory arrays. There are two main counters which are used for searching process. "Count1" is used for searching character in dictionary. If character is present in dictionary then its code is saved in other buffer that is "de11". "Count" buffer is shifted to next value and start to point next character present in input data. All searched characters will save in "check" buffer. Once the content of check buffer is equal to content of "data read" buffer then searching process indicate to completed and their codes will save in "de11" buffer which is shown in figure 5compressed output isgenerated through file shown in figure 7.

Input text - connect the input to logic one \& two \& three.

Outputtext-12114514615730171419514251522372152315551482

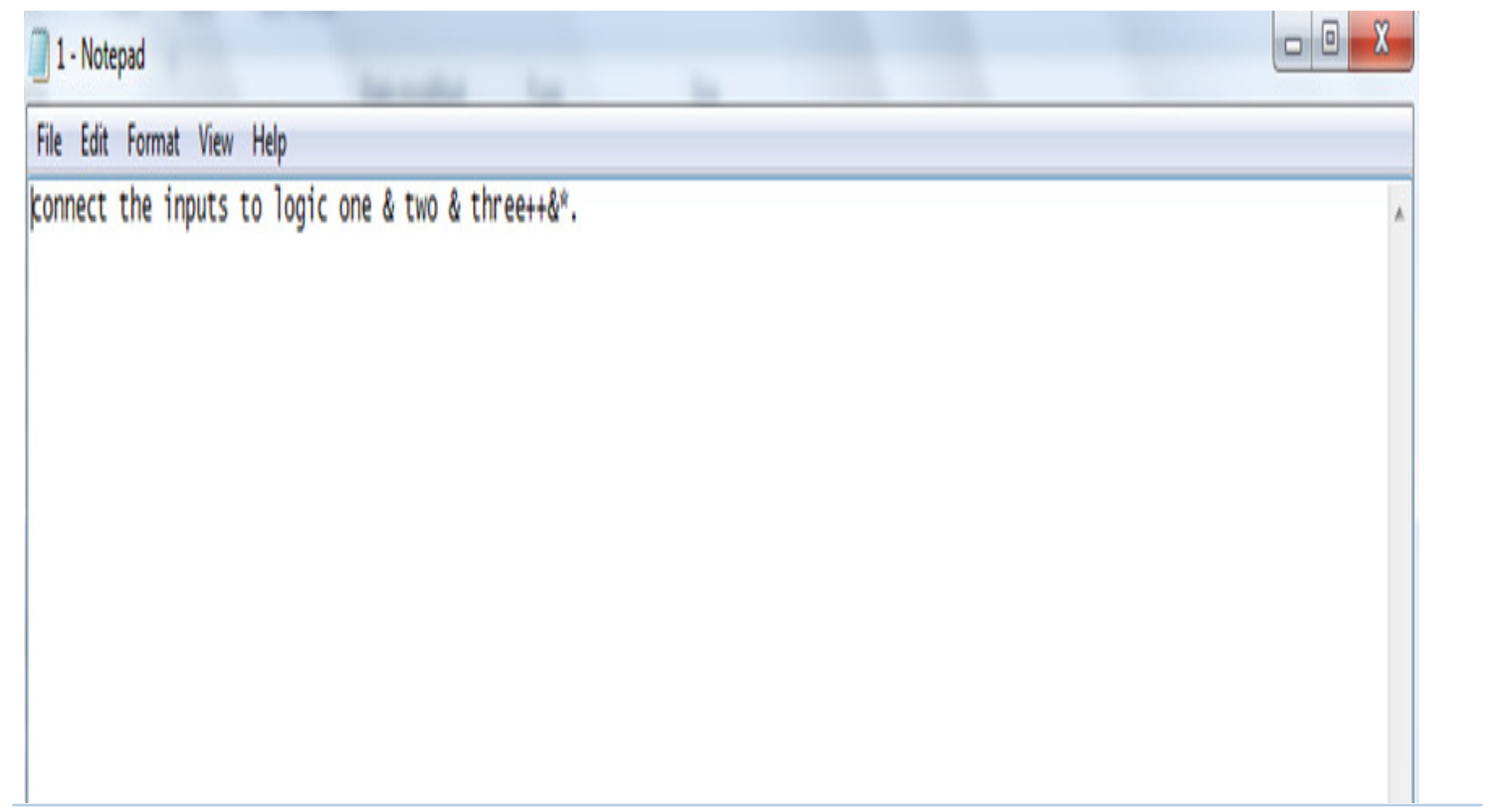

Figure 4: Enter data through file which we want to compress

Given Input text - connect the input to logic one \& two \& three 
International Journal of Information Sciences and Techniques (IJIST) Vol.2, No.4, July 2012

Input is given by text file which is one of the data type of vhdl language. File is used for giving input as a collection of characters in one clock cycle.

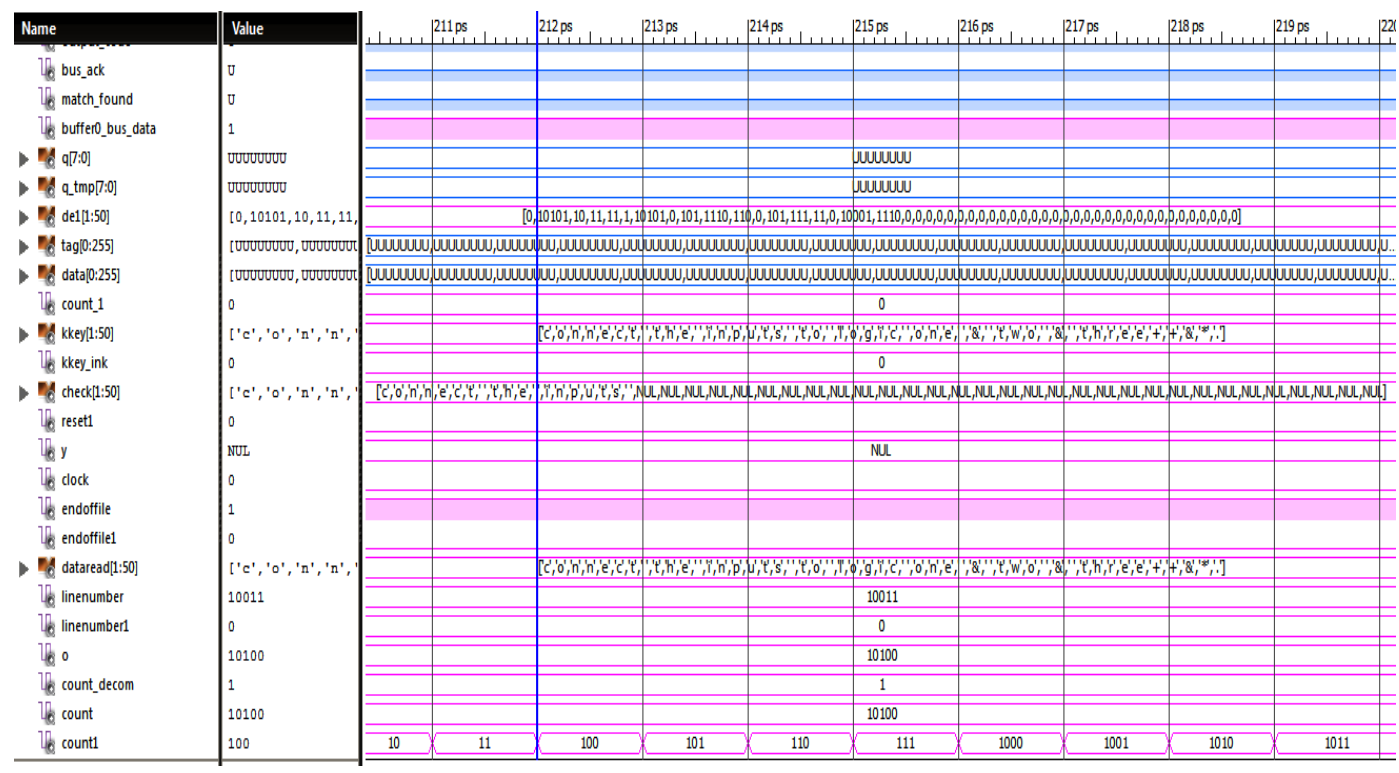

Figure 5: Searching process (Searching each character from dictionary)

Simulation for LZW Compression algorithm observed on Xilinx tool. When 350 bits entered to LZW compression algorithm then it is transmitted to 119 bits and clock rate for simulation is 493 ps.

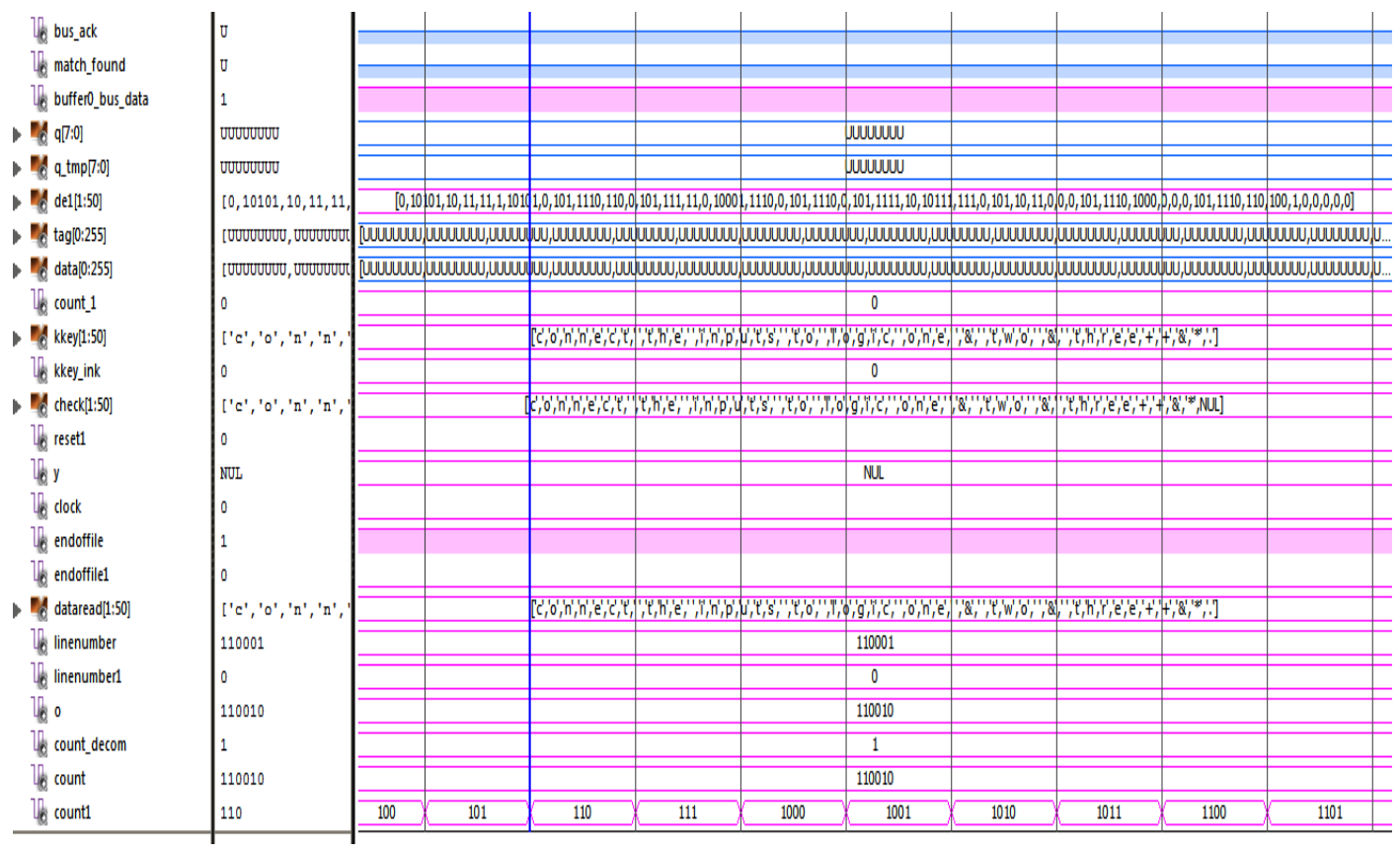

Figure 6: Complete data compression process 
International Journal of Information Sciences and Techniques (IJIST) Vol.2, No.4, July 2012

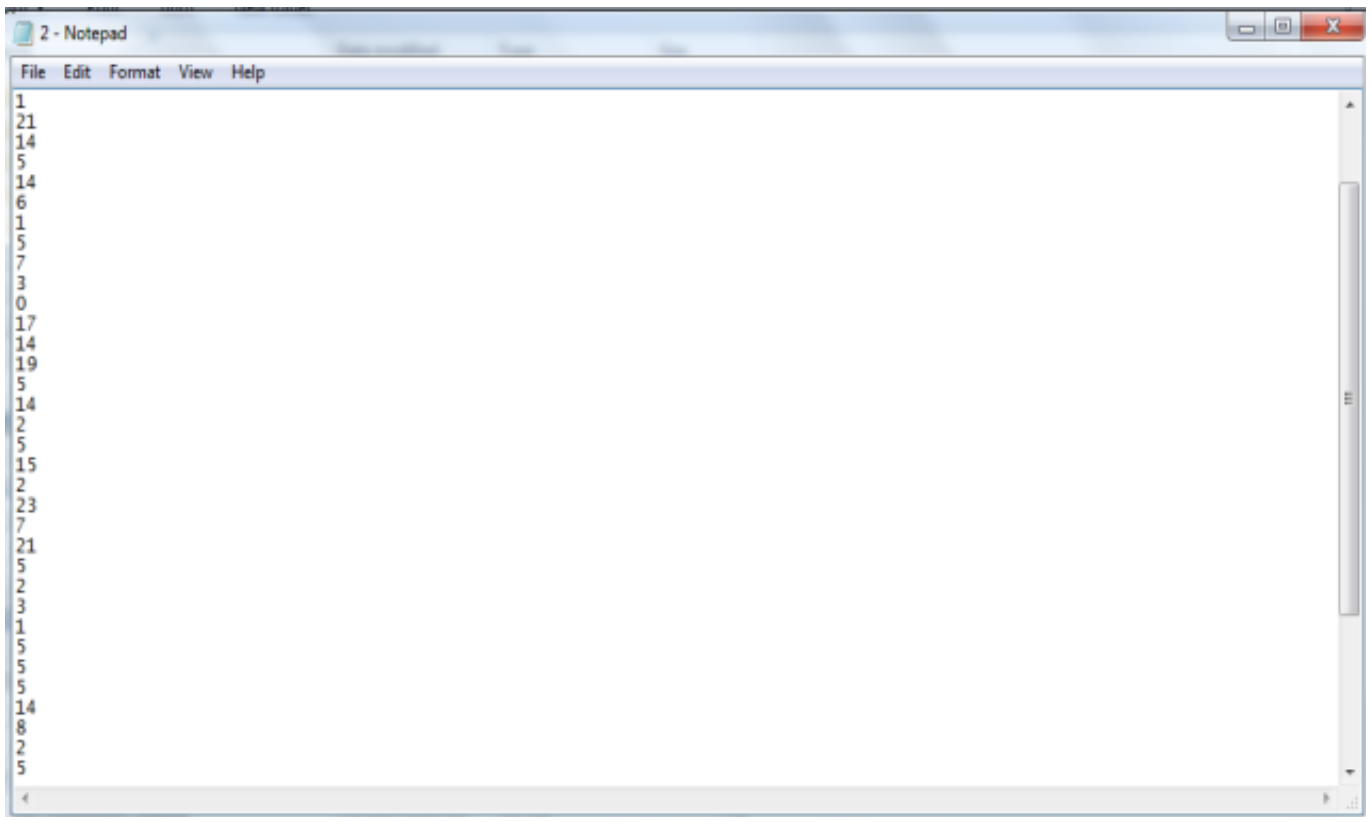

Figure 7: Compressed output generate on file

Output text-12114514615730171419514251522372152315551482

\subsection{RTL view of LZW Compressor}

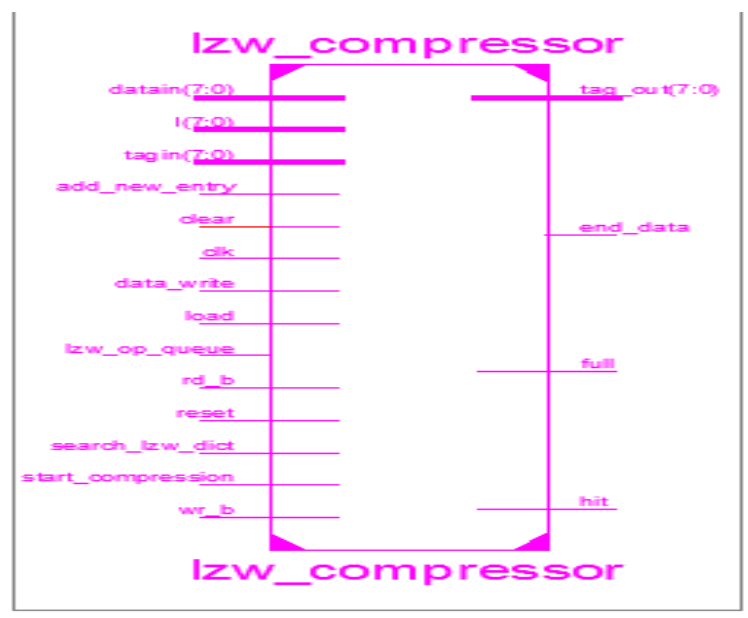

Figure-8: RTL view of LZW Compressor

This RTL view shows the signals which are used for proposed LZW data compression algorithm. Reset, clock, start_compression used for initialization of data compression. Load, data_write, wr_b, rd_b are signals used for buffer in LZW algorithm. Search_lzw is for searching data in dictionary. The signal description of this proposed algorithm is shown in table 2 . 
International Journal of Information Sciences and Techniques (IJIST) Vol.2, No.4, July 2012

\subsubsection{Signal description of LZW Compressor}

Table 2: Input/output signals with Remarks

\begin{tabular}{|l|l|}
\hline Name & \multicolumn{1}{|c|}{ Description } \\
\hline Reset & To reset \\
\hline Clock & Provide clock \\
\hline Start_compression & Signal for start compression \\
\hline Data_write & Signal for write data \\
\hline Load & For data load in buffer \\
\hline Clear & Clear buffer \\
\hline Wr_b & Signal for write and read \\
\hline Rd_b & Signal for write and read \\
\hline Search_lzw & For searching \\
\hline Add_new_entry & For adding new data \\
\hline Data_in & Enter value \\
\hline
\end{tabular}

\subsubsection{Analysis of compression rate with different bit size}

Table 3: Analysis of compression rate

\begin{tabular}{|c|c|c|c|}
\hline Word Size & Compression Rate & $\begin{array}{c}\text { Compressed Bit } \\
\text { Size }\end{array}$ & Original Bit Size \\
\hline 4 & 53.125 & 17 & 28 \\
\hline 10 & 33.75 & 27 & 70 \\
\hline 38 & 30.11 & 95 & 265 \\
\hline 50 & 29.75 & 119 & 350 \\
\hline
\end{tabular}

\subsubsection{Verification and Synthesis}

For system verification, we successfully execute proposed LZW algorithm. Test case for finite state machine is generated in VHDL. The synthesis result of LZW compression algorithm is summarized in table 4 . The synthesis report shows device utilization summary.

Table 4: Device Utilization Summary

\begin{tabular}{|l|l|}
\hline Number of Slices & 3606 out of $614458 \%$ \\
\hline Number of Slice Flip Flops & 4097 out of $1228833 \%$ \\
\hline Number of 4 input LUTs & 4190 out of $1228834 \%$ \\
\hline Number of IOs: & 30 \\
\hline Number of bonded IOBs: & 30 out of $240 \quad 12 \%$ \\
\hline IOB Flip Flops: & 1 \\
\hline
\end{tabular}




\subsection{Comparison of the Results with the Previous Work}

The results achieved are compared with the reference work is shown in table 5. It is concluded that enhancement in the performance of LZW Data Compression algorithm by using less number of bits than their ASCII code, utilizing content addressable memory arrays. Thus the text data can be effectively compressed and compared with previous work. In addition to this, the proposed research work, results show the reduction in storage space by $60.25 \%$ and increase the compression rate by $30.3 \%$. Comparison of this work with previous work is described in table 5 shown as:

Table 5: Comparison between this research works with previous work

\begin{tabular}{|c|c|c|}
\hline Input Size & Compressed bit with previous work & $\begin{array}{c}\text { Compressed bit with } \\
\text { Improved LZW }\end{array}$ \\
\hline 112 & 104 & 70 \\
\hline 144 & 96 & 90 \\
\hline 152 & 100 & 95 \\
\hline 184 & 156 & 115 \\
\hline 360 & 296 & 225 \\
\hline
\end{tabular}

\section{Conclusions}

In order to get better compression rate, the proposed dictionary based LZW algorithm can replace their codes with 5 bits instead of 7 bits ASCII code. LZW algorithm is evaluated by finite state machine technique. With this technique we have observed that storage space is reduced up to $60.25 \%$ and compression rate improved up to $30.3 \%$. Weanalyze compression rate with different number of input bits on Xilinx tool.

\section{References}

[1] Parvinder Singh,ManojDuhan and Priyanka(2006)“Enhancing LZW Algorithm to Increase Overall Performance", Annual IEEE Indian Conference,pp1-4.

[2] Ming-Bo Lin, Jang-Feng Lee, G. E. Jan,( 2006)“ALossless Data Compression and Decompression Algorithm and Its Hardware Architecture” VLSI IEEE Transactions ,Vol.14,pp925-936.

[3] YiCao, Guoging Wu, Huawei Wang,( 2011) "A Smart Compression Scheme for GPU-Accelerated Volume Rendering of Time-Varying Data." Virtual Reality and Visualization (ICVRV) conference.pp205-210

[4] Guolv.Tan, Yujun Wang,( 2009) "A Compression Error and Optimize Compression Algorithm for vector Data.", Environmental Science and Information application technology,vol.2,pp522-525.

[5] Parvinder Singh, Sudhir Batra, and HR Sharma,( 2005) "Evaluating the performance of message hidden in 1st and 2nd bit plane", WSEAS Trans. on Information Science an Applications, vol 2, pp $1220-1227$.

[6] Ozsoy, A. Swany, "LZSS Lossless Data Compression on CUDA",( 2011) "IEEE international conference on Cluster computing (CLUSTER),pp403-411.

[7] Mateosian, R, "Introduction to Data Compression" (1996), vol.16.

[8] Henriques and N. Ranganathan,(2005) "A parallel architecture for data compression," IEEE Symp .on parallel and distributed processing Parallel. Distribution, pp260-266. 
International Journal of Information Sciences and Techniques (IJIST) Vol.2, No.4, July 2012

[9] Huan Zhang, Xiao-ping Fan, Shao-qiang Liu Zhi Zhong "Design and Realization of Improved LZW Algorithm for Wireless Sensor Networks",International Conference on Information Science and Technology,pp671-675.

\section{Bibliographies}

Simrandeep Kaur received the B.Tech degree in Computer Science Engineering from the Punjab technical university, Punjab in 2010, and pursuing M.Tech degree in VLSI Design from Centre of Development and Advance Computing Mohali, Punjab .Currently, she is doing her thesis work on data compression technique. Her topic of interest is data compression, security system, data structure and embedded system.Emailsimrandeepkaur25@yahoo.com

VemuSulochana has obtained her Bachelor of Technology degree in Electronics \& Communication Engineering from JNTU Kakinada and Master of Technology degree in VLSI Design Automation \& Techniques from NIT, Hamirpur in 2004 and 2009 respectively. She is working as a Project consultant at C-DAC, Mohali to conduct innovative research in the area of VLSI design. Her research interests include low power VLSI design, Computer-aided design (CAD), Digital \& Analog VLSI Desig n. She enjoys teaching VLSI design, Device modelling, Low-power VLSI Design, Analog \& mixed sign al VLSI Design. Email-id is suchivlsi@gmail.com
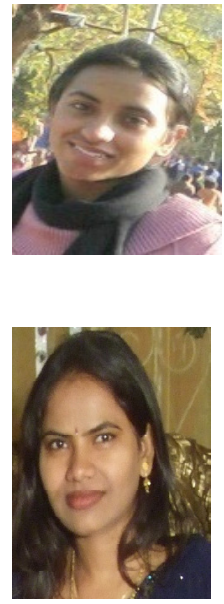